\title{
Automated Optical Inspection of Solder Paste based on 2.5D Visual Images
}

\author{
Grantham K.H. Pang and Ming-Hei Chu \\ Department of Electrical and Electronic Engineering, \\ Industrial Automation Research Laboratory, \\ The University of Hong Kong, Pokfulam Road, Hong Kong \\ gpang@eee.hku.hk
}

\begin{abstract}
In this paper, a special technique for the inspection of solder paste using directional LED lighting is presented. Conventional optical inspection method would depend on an image acquired from a camera mounted from the top. This 2D inspection of solder paste based on images is fast but is limited to defect such as bridge or no solder. Defects related to the volume of the printed solder paste or unevenness of the paste cannot be treated from a top image. The developed technique of this paper would involve the use of special directional side lighting to acquire two-and-a-half dimensional (2.5D) images from above the solder paste block. A sequence of three images is acquired and image processing is carried out for defect detection of the printed solder paste. The acquired images would highlight the geometrical features of the solder paste block. Solder paste inspection is then carried out based on the highlighted features. The proposed method can handle other types of defects that cannot be treated by conventional top light images.
\end{abstract}

Index Terms - Process automation, surface mount technology manufacturing, solder paste printing inspection, fuzzy system.

\section{INTRODUCTION}

During the manufacturing of printed circuit board (PCB), the very first stage is the deposit of solder paste pads onto the PCBs. The height of the pads can be obtained based on laser triangulation. However, the inspection speed would be slow and very often cannot meet the requirement of an in-line process. Also, a laser scanning system would cost over USD $400 \mathrm{~K}$ and is very expensive.

Inspection at an early stage would ensure that the specified amount of paste is deposited correctly on the pads. With the right amount of paste, it allows for a greater margin of error in the subsequent component placement and reflow processes. From some study, it has been found that almost $40 \%$ of defects can be due to poor solder paste printing. Such defects include bridge, bad joints/connection after reflow due to insufficient solder, off registration and excess solder. In fact, the volume of solder paste also affects the quality of the final product. Low solder paste volumes can

Grantham .K.H. Pang is with the Department of Electrical and Electronic Engineering, The University of Hong Kong (e-mail: gpang@eee.hku.hk). produce solder joints that pass electrical test but have low mechanical strength and high failure rates. It must be mentioned that not all the end defects are due to the solder paste printing process. However, early defect detection can eliminate many subsequent defects and improve the yield. Also, if defects can be detected immediately after solder paste printing, the repair cost would be the lowest.

The inspection of solder paste using laser is expensive. In laser triangulation, a spot of light from a laser diode is pointed onto the surface of the object under inspection. The reflected laser enters into the window of a light sensitive digital detector array. The height of a point of the object can then be found by triangulation. In practice, a line of laser is projected instead of a laser spot. When the projecting device moves across the object, a camera is used to capture a number of images at even time interval. If high resolution is required, then more images (and also measurement time) are required, which is a time consuming method. This technique involves high-cost machine and not used in a typical production line.

Solder paste inspection in an SMT line usually involves the acquisition of a color image of the printed solder paste blocks under top lighting. Defects such as bridge would appear as a connection between neighboring blocks. No solder would also appear well for the color properties of an area without solder should be quite different from the solder paste. However, defects related to insufficient solder, printed volume or the evenness of the solder paste would not be detected. This paper aims to address on these issues and develops a new method based on the use of directional lighting. A sequence of images are acquired with the use of side light from different directions. Special 2.5D visual images would highlight the edges, and these edge information would help to detect for the defects.

\section{REVIEW OF ADVANCED SOLDER PASTE INSPECTION METHODS}

The method for solder paste inspection has been based on constructing the 3D surface model. The approaches include the followings: 
- Laser triangulation method,

- Shape From Shading (SFS) method,

- Phase Profilometry method using structured light source,

- Reconstruction by Neural Network.

The laser method aims to obtain the height of the solder paste by measuring point-by-point or line-by-line the height data by a laser head sensor. The calculation is based on triangulation and the height data of the solder paste is then used for the reconstruction of the $3 \mathrm{D}$ profile. Many commercial systems [1] have been developed based on this approach. Accurate measurement can be obtained. However, such laser scan systems are typically very expensive and have low inspection speed.

The first shape-from-shading (SFS) technique was developed by Horn in the early 1970s [2]. The technique is based on recovering the shape from a gradual variation of shading in the image. In SFS, the aim is to recover the light source and the surface shape at each pixel in the image. Zhang et al. [3] have examined other approaches that have emerged and compared their performance. It has been found that none of the six well-known SFS algorithms compared has consistent performance for all test images. They all work well for certain images, but perform poorly for others. Also, the processing time typically required by the SFS algorithms is quite long.

In phase profilometry, a structured light pattern (usually grayscale fringes) is projected onto the object and viewed by a camera. The imaged pattern is phase-modulated according to the topography of the object. The extraction of the phase information enables the reconstruction of the 3D shape of the object [4]. The Fourier transform profilometry [5] relies on processing the image in the frequency domain. Another method relies on processing the image in the real signal domain [6]. In [7], a binary light pattern, Ronchi Pattern, was proposed instead of the traditional grayscale fringe pattern. The Ronchi Pattern can save the space of the light sources and the binary pattern can help to avoid issues like image saturation and specular nature of the imaged surface. A distinct binary code was given to each surface point through a number of physical shifting of the fringe grating and the image data. The height data is then obtained based on correspondence establishment between fringe grating plane and image plane. One drawback of the work is that only the height data at the edge points in the image data can be constructed. The 3D information of non-edge points have to be interpolated from the edge points.

On 3D reconstruction by neural network, an ANN automatic optical inspection model is proposed in [8]. The input nodes of the ANN model consist of the image features that are captured from images using different light sources. The output nodes are the heights of the corresponding image pixel areas. The training patterns of the model use the laser
$3 \mathrm{D}$ inspection results. It is claimed that the estimated 3D surface model achieves $90 \%$ accuracy on average.

In another method [9-11], researchers have used neural network to solve problem in SFS, which is then used for 3D shape reconstruction. Wei et al. [9] have used a multilayer neural network approach to tackle the SFS problem. Cho and Chow [10-11] have developed more sophisticated ANN models. In [11], new neural-based reflectance models are presented. The feedforward neural network (FNN) model is able to generalize the diffuse term and the radial-basis function (RBF) model is able to generalize the specular term. Also, a hybrid structure of FNN-based model and RBF-based model is presented because most real surfaces are neither Lambertian models nor ideally specular models. In [12], a preliminary idea on solder paste inspection based on directional lighting was given. This paper is a novel development from that work. The new method makes use of colored lighting, and the number of acquired images is three, instead of five in [12].

\section{2.5D VISUAL IMAGES FOR SOLDER PASTE INSPECTION}

The developed method would involve the acquisition of images with directional LED lighting. The colored side lighting is designed to highlight the 3D information of the solder paste blocks. Images of solder paste block are captured and processed by an Image Acquisition Module. Then, an Image Processing Module would extract the highlighted features and the information can be passed to a subsequent module to calculate a quality score on the solder paste block. In this paper, the focus is on the Image Acquisition and Processing Modules. The system setup of the proposed methodology involves various hardware including high resolution digital camera, LED lighting, lighting controller, $\mathrm{X}-\mathrm{Y}$ table and computer. Figure 1 shows the hardware required for the Image Acquisition Module.

\section{$\underline{\text { Lighting Design }}$}

A solder paste block is illuminated with three consecutive illumination patterns: side north-south, side east-west and top lighting. Red and blue LED lightings are used in the design. Fig. 2 shows a cross-sectional view of a typical solder paste block under the designed Red-Blue lighting.

A red side light and a blue side light are applied in opposite directions at an angle of around 40 degrees with reference to the horizontal plane. 


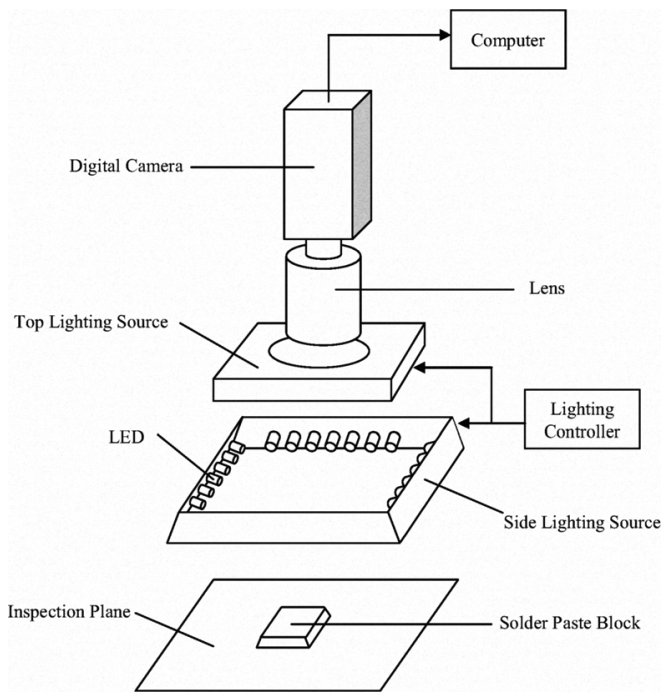

Fig. 1 Side light images of solder paste blocks

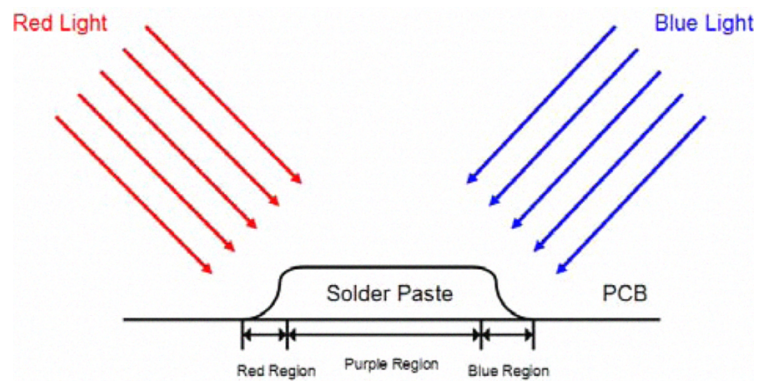

Fig. 2 Side view of the solder paste block

Under the designed illumination, the horizontal surface on the solder paste block is highlighted by a mixture of red and blue lights and would appear purple in color. The inclined surface would be highlighted by the light which the inclined surface faces.

Next, the solder paste block is illuminated by even top lighting. The top light makes use of white LED to provide illumination. The aim of the top lighting is to locate the position of the solder paste block and extract its size. Using the top lighting, a top view of the actual edge boundaries of the solder paste block can be extracted. The flux surrounding the solder paste block would not be visible from the top light image. Together with the mask information of the block using the top lighting image, we can check whether the solder paste block contains defects. With further processing of the edge pixels, a quality score may be computed.

For each illumination, an image is taken by a digital camera for subsequent image processing. Fig. 3 gives the photo of the experimental setup.

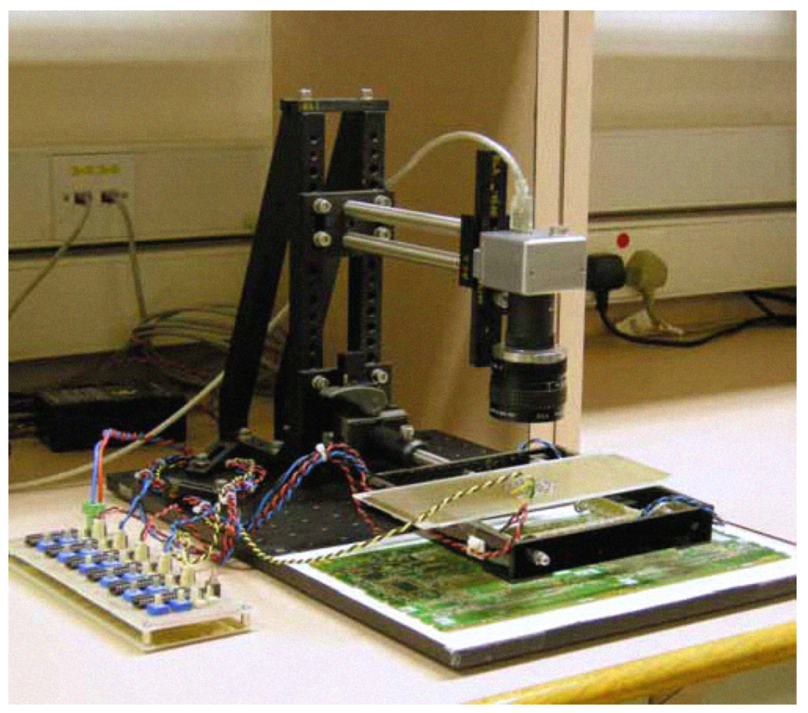

Fig. 3 Photo of the experimental setup

To provide the kind of illumination pattern in Figure 2, the LED arrangement in the side lighting source is shown in Figure 4 below:

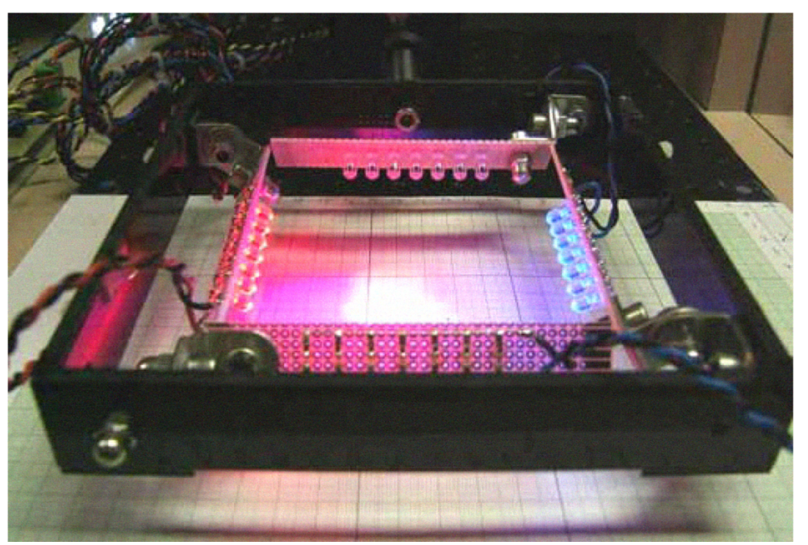

Fig. 4 LED arrangement in side lighting

In the side lighting source, there are four LED sources north, south, east and west lighting. Blue LEDs are used in north and west lighting whereas red LEDs are used in south and west lighting. The side lighting source provides two different lighting patterns - north-south illumination and east-west illumination. These are 2.5D images which contain three dimensional shape information in a two dimensional image. Together with the top lighting source 
which gives the top light illumination, there are totally three illumination patterns used in the methodology.

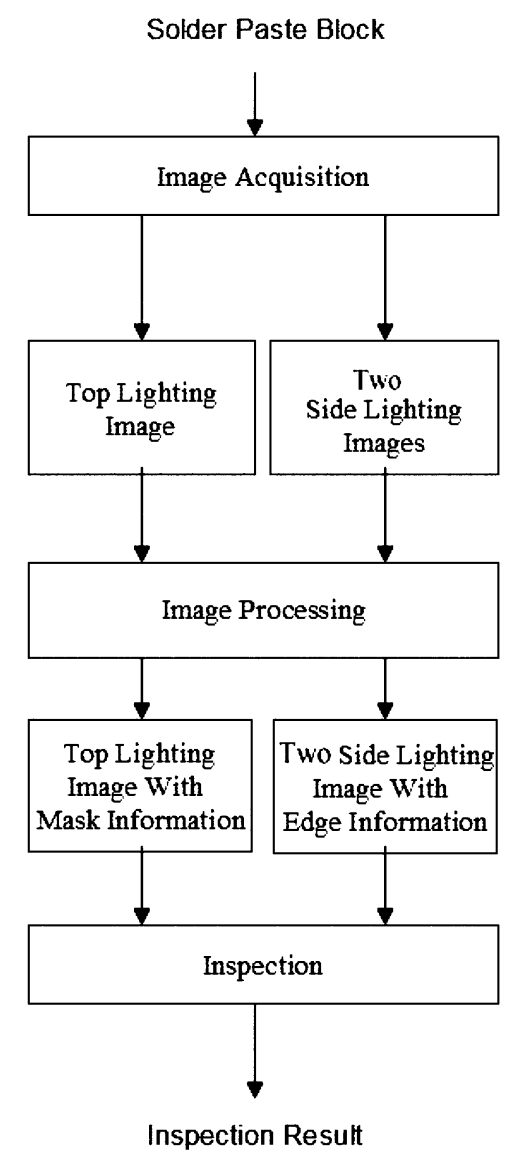

Fig. 4 Block diagram of the developed method

\section{EXPERIMENTAL HARDWARE}

The Industrial Automation Research Laboratory has developed an AOI machine for 3D inspection of solder paste The current machine is composed of three linear actuators (two for the $\mathrm{Y}$-axis and one for the $\mathrm{X}$-axis) and is essentially a high accuracy $X-Y$ table mounted with an RGB 1280x960-pixel CCD camera. The position accuracy achieves $0.04 \mathrm{~mm}$. With a field-of-view (FOV) of $23 \mathrm{~mm} \mathrm{x}$ $17.25 \mathrm{~mm}$, the resolution is $1415 \mathrm{dpi}$. Photo of the hardware of the developed inspection machine in the Industrial Automation Research Laboratory of the Dept. of Electrical and Electronic Engg. of The University of Hong Kong is given in Figure 5.

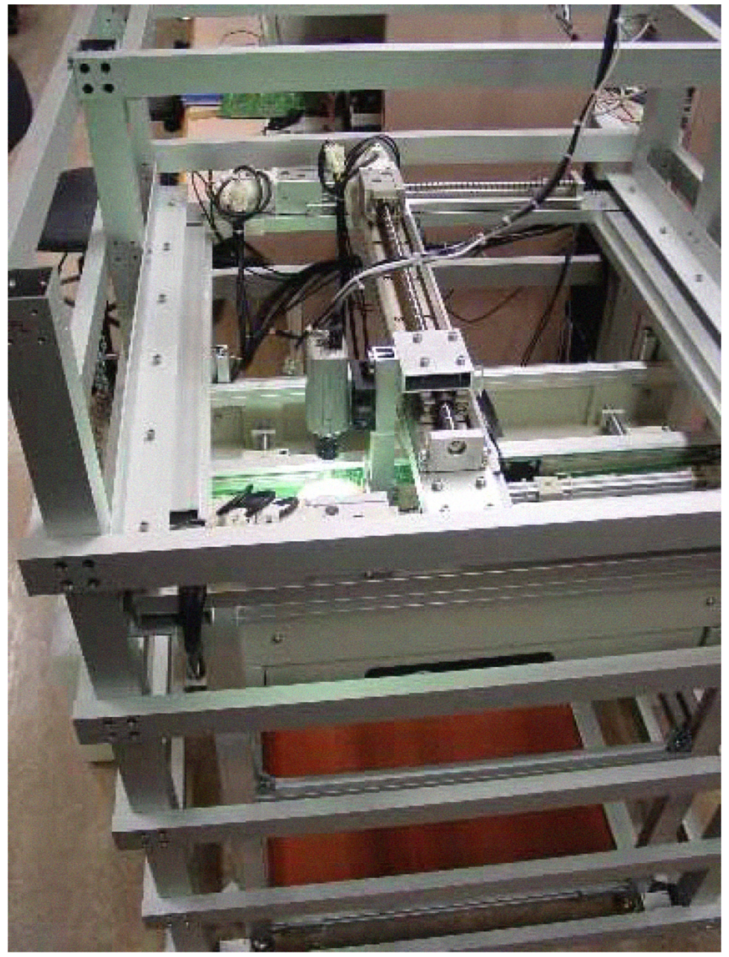

Fig. 5 Developed Inspection Machine

\section{ILLUMINATION PATTERN OF 2.5D IMAGES}

Fig. 6(a) shows a typical solder paste block under side east-west lighting. The same block under side north-south lighting is shown in Fig. 6(b).
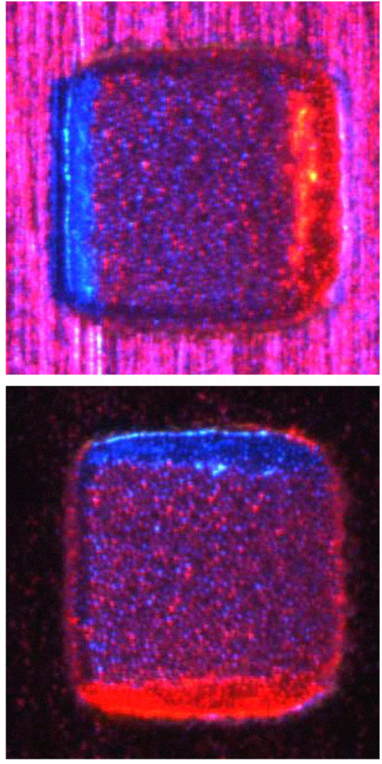

Fig. 6 Images of a solder paste block without defect under (a) east-west lighting (b) north-south lighting 
Figure 7 shows the two acquired 2.5D images of a solder paste block with the defect of insufficient solder, under two side-light illuminations.
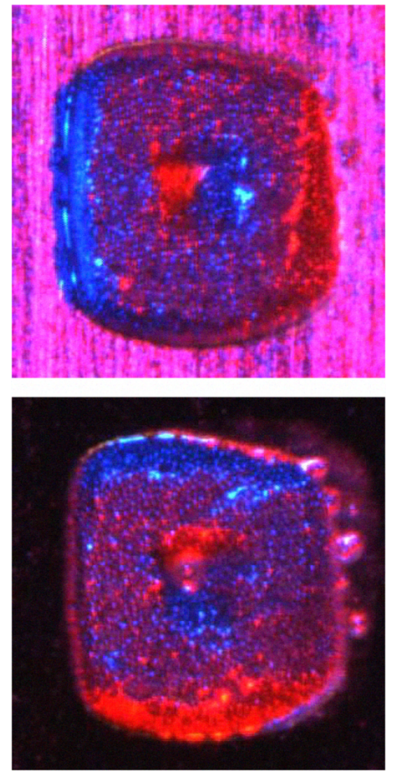

Fig. 7 Images of a solder paste block with defect of insufficient solder under (a) east-west lighting (b) north-south lighting

Figure 8 gives another example of a solder paste block, which has excess solder in the center area of the block.
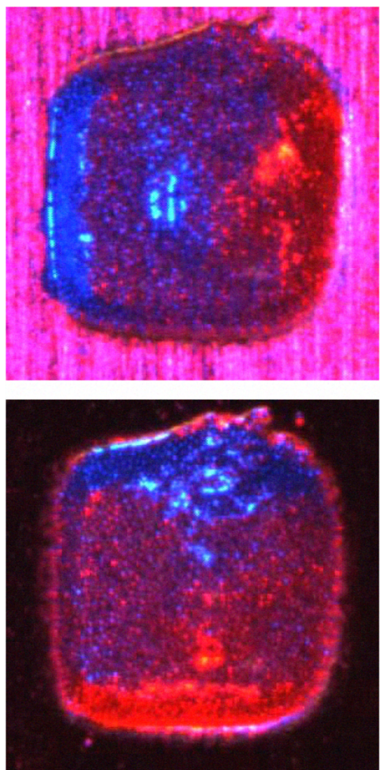

Fig. 8 Images of a solder paste block with defect of excess solder under (a) east-west lighting (b) north-south lighting

Again, the 2.5D images have highlighted the defect and enable its detection in subsequent image processing.

\section{IMAGE PROCESSING}

The directional, colored, side LED lighting can effectively highlight the geometrical features of the solder paste block. Under the designed lighting, inclined surfaces in the solder paste block appear red or blue color in the captured images. These highlighted inclined surfaces can be used for the inspection of solder paste block. To facilitate the inspection process, they should be extracted from the captured image. Image processing techniques are used to extract these highlighted surfaces in the image. Below are examples of processing images for three different types of solder paste blocks.

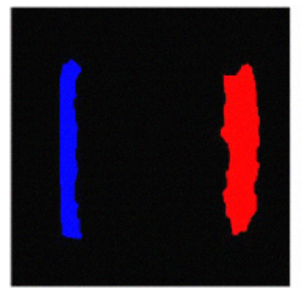

(a) Without defect

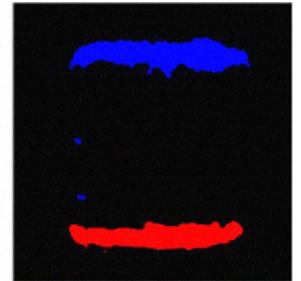

(b)

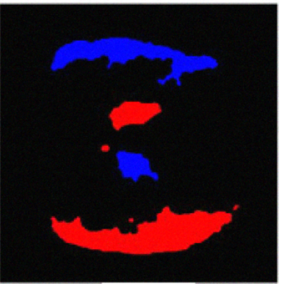

(d)

(c) With defect (Insufficient solder)
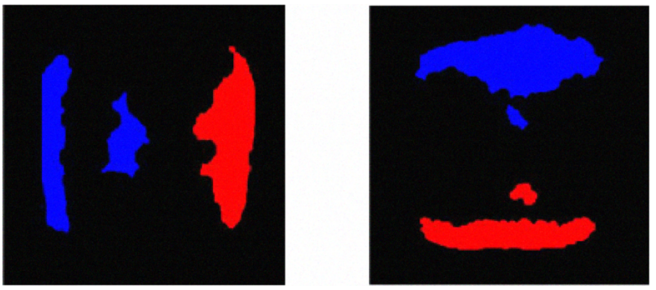

(e) With defect

(Excess solder)
Fig. 9 Color clusters extracted from three different types of solder paste blocks

It can be observed that colored clusters would appear between the two edge regions, and the location of those clusters would also give an indication to the defect type. The size of those colored regions would also indicate the severity of the defect, or the defect type. From an analysis, twenty features of the solder paste block are extracted and their descriptions are given as follows:

Feature 1 West Edge Thickness

By calculating the average width of all the rows of blue pixels in the West Edge Region

Feature 2 East Edge Thickness 
By calculating the average width of all the rows of red pixels in the East Edge Region

Feature 3 North Edge Thickness

By calculating the average width of all the columns of blue pixels in the North Edge Region

Feature 4 South Edge Thickness

By calculating the average width of all the columns of red pixels in the South Edge Region

Feature 5 West Edge Thickness Deviation

By calculating the standard deviation of the West Edge Thickness

Feature 6 East Edge Thickness Deviation

By calculating the standard deviation of the East Edge Thickness

Feature 7 North Edge Thickness Deviation

By calculating the standard deviation of the North Edge Thickness

Feature 8 South Edge Thickness Deviation

By calculating the standard deviation of the South Edge Thickness

Feature 9 West Edge pixels' connectivity By counting the number of gaps vertically in the West Edge Region

Feature 10 East Edge pixels' connectivity.

By counting the number of gaps vertically in the East Edge Region

Feature 11 North Edge pixels' connectivity

By counting the number of gaps horizontally in the North Edge Region

Feature 12 South Edge pixels' connectivity

By counting the number of gaps horizontally in the South Edge Region

Feature 13 West Edge Region Surface Area

By calculating the number of red pixels in the West Edge Region

Feature 14 East Edge Region Surface Area

By calculating the number of blue pixels in the East Edge Region

Feature 15 North Edge Region Surface Area

By calculating the number of blue pixels in the North Edge Region

Feature 16 South Edge Region Surface Area

By calculating the number of red pixels in the South Edge Region

Feature 17 Central Region Surface Area

By calculating the number of pixels within the boundaries of the Central Region

Feature 18 Central Region Defect Area

By calculating the number of red and blue pixels within the Central Region

Feature 19 Solder Paste Block Shifting

By calculating the horizontal and vertical shift of the block with respect to a reference location

Feature 20 Solder Paste Surface Area

By calculating the number of pixels within the block as seen from the top light image

\section{CONCLUSIONS}

In this paper, the Image Acquisition Module and Image Processing module of a novel $2.5 \mathrm{D}$ visual inspection method for solder paste is presented. The developed method involves the use of special directional LED side lighting on the solder paste blocks. A sequence of two 2.5D images plus one top light image is acquired and image processing is carried out for the edge information of the printed solder paste.

The special technique also involves other modules for feature extraction from the processed image, and a fuzzy system for calculating a quality score which would indicate the printing quality of the solder paste block. Neural-fuzzy training can also be applied to the fuzzy system for fine-tuning the behavior according to the users' preference. For details on the design of those modules, the reader can refer to [12].

\section{ACKNOWLEDGEMENTS}

This research is supported by a grant from the Research Grant Council of Hong Kong (HKU 7163/06E).

\section{REFERENCES}

[1] Jeffrey Rupert, Doreen Tan and Pat Pilon, "3D Inspection for the measurement of solder paste deposits", APEX 2000, March 14-16, 2000.

[2] B.K.P. Horn, "Shape from Shading: A Method for Obtaining the Shape of a smooth Opaque Object from One View", Ph.D. thesis, Massachusetts Instituteof Technology, 1970.

[3] Ruo Zhang; Ping-Sing Tsai; Cryer, J.E.; Shah M. "Shape From Shading: A Survey", IEEE Transactions on Pattern Analysis and Machine Intelligence Volume 21, Issue 8, pp. 690-706, Aug. 1999.

[4] Luigi Di Stefano and Frank Boland, "A new phase extraction algorithm for phase profilometry", Machine Vision and Applications, Volume 10, Number 4, pp. 188 - 200, December 1997.

[5] M. Takeda and K. Mutoh, "Fourier transform profilometry for the automatic measurement of 3-D object shapes", Applied Optics, 22 (24), pp. 3977-3982, 1982.

[6] S. Tang and Y. Hung, "Fast profilometer for the automatic measurement of 3-D object shapes", Applied Optics, 29 (20), pp. 3012-3018, 1990

[7] Jun Cheng, Ronald Chung, Edmund Y. Lam, Kenneth S.M. Fung, Fan Wang, and W.H. Leung, "Three-dimensional reconstruction of wafer solder bumps using binary pattern projection," in Machine Vision Applications in Industrial Inspection XIII, vol. 5679 of Proceedings of the SPIE, pp. 44-52, January 2005.

[8] Fang-Chung Yang, Chung-Hsien Kuo, Jein-Jong Wing, Ching-Kun Yang, "Reconstructing the 3D solder paste surface model using image processing and artificial neural network", 2004 IEEE International Conference on Systems, Man and Cybernetics, Volume 3, 10-13, pp. 3051 - 3056, Oct. 2004.

[9] G.Q. Wei and G. Hirzinger, "Learning shape from shading by a multilayer network", IEEE Trans. Neural Networks, Vol. 17, pp. 985-995, 1996.

[10] S. Y. Cho and T. W. S. Chow, "Shape recovery from shading by a new neural-based reflectance model", IEEE Trans. Neural Networks, Vol. 10, pp. $1536-1541,1999$.

[11] S.Y. Cho and T.W. S. Chow, "Neural Computation Approach for Developing a 3-D Shape Reconstruction Model", IEEE Transactions on Neural Network, Vol. 12, No. 5, pp. 1204-1214, September 2001.

[12] G.K.H. Pang, and M. Chu, "Automated Inspection of Solder Paste by Directional LED Lighting", IEEE International Conference on Automation and Logistics, Qingdao, P.R. China, Sept 1-3, 2008. 